Original paper

\title{
Pemafibrate improves hepatic inflammation, function and fibrosis in patients with non-alcoholic fatty liver disease: a one-year observational study
}

\author{
Satoshi Shinozaki ${ }^{1,2}$, Toshiyuki Tahara ${ }^{3}$, Alan Kawarai Lefor ${ }^{4}$, Masahito Ogura ${ }^{5}$ \\ 'Shinozaki Medical Clinic, Japan \\ 2Department of Medicine, Division of Gastroenterology, Jichi Medical University, Japan \\ ${ }^{3}$ Saiseikai Utsunomiya Hospital, Japan \\ ${ }^{4}$ Department of Surgery, Jichi Medical University, Japan \\ ${ }^{5}$ Department of Diabetes, Endocrinology and Nutrition, Graduate School of Medicine, Kyoto University, Japan
}

\begin{abstract}
Aim of the study: To optimize the long-term outcomes of patients with non-alcoholic fatty liver disease (NAFLD), long-term therapy is important to prevent cirrhosis and hepatocellular carcinoma. Pemafibrate, a novel selective peroxisome proliferator-activated receptor- $\alpha$ modulator, is a promising therapeutic agent for patients with NAFLD. However, only short-term clinical studies are currently available. The aim of this study is to evaluate the long-term outcomes of patients with NAFLD treated with pemafibrate.

Material and methods: This is a retrospective observational study. Patients with NAFLD treated with pemafibrate $0.1 \mathrm{mg}$ twice daily for one year were retrospectively reviewed.

Results: Twenty-two patients without diabetes mellitus were included and analyzed. Regarding hepatic inflammation markers, alanine aminotransferase (ALT) significantly decreased during the first three months and was maintained. Low-density lipoprotein and triglycerides significantly decreased at three months and were maintained. Regarding markers of hepatic function, the albumin-bilirubin score decreased significantly during one year of therapy due to significantly elevated serum albumin and decreased total bilirubin levels. Regarding markers of fibrosis, Mac-2 binding protein glucosylation isomer (M2BPGi) significantly decreased, and platelet count increased significantly. Next, we performed correlation analysis between changes in M2BPGi and other parameters. Changes in aspartate aminotransferase, ALT and triglycerides positively correlated with the change in M2BPGi.

Conclusions: One-year pemafibrate therapy improves markers of hepatic inflammation, function and fibrosis in non-diabetic patients with NAFLD. Improvement of hepatic fibrosis markers significantly correlates with improvement of hepatic inflammation markers and triglyceride levels.
\end{abstract}

Key words: non-alcoholic fatty liver disease, non-alcoholic steatohepatitis, pemafibrate, dyslipidemia, hepatitis.

Address for correspondence:

Dr. Satoshi Shinozaki, Shinozaki Medical Clinic, Department of Medicine, Division of Gastroenterology, Jichi Medical University, Japan, e-mail: shinozaki-s@aqua.ocn.ne.jp

\section{Introduction}

Non-alcoholic fatty liver disease (NAFLD) is a hepatic manifestation of metabolic syndrome and is characterized by hepatic steatosis and inflammation resulting in fibrosis [1]. NAFLD is divided into non-alcoholic fatty liver and non-alcoholic steatohepatitis (NASH), and NASH has the potential to progress to cirrhosis and/or hepatocellular carcinoma. However, no specific treatment for NAFLD has been approved. Weight control is a viable option, but $10 \%$ or more weight reduction is difficult in most patients [2]. Due to a decrease in 
the incidence of viral hepatitis due to the development and dissemination of direct acting drugs, NAFLD is expected to be the main cause of hepatocellular carcinoma and cirrhosis in the near future. Long-term control of inflammation and fibrosis caused by NAFLD is important to prevent cirrhosis and hepatocellular carcinoma.

Pemafibrate, a novel selective peroxisome proliferator-activated receptor- $\alpha$ modulator (SPPARM $\alpha$ ), was developed and disseminated in Japan for the treatment of dyslipidemia. The protective effect of pemafibrate against NAFLD was shown in a murine model of NASH [3]. In clinical studies, a phase II trial demonstrated improvement in lipid profiles and liver enzymes in patients without the diagnosis of NAFLD [4]. We previously reported the short-term outcomes of patients with NAFLD treated with pemafibrate that showed significant improvement in markers of hepatic inflammation and function at three months [5]. Existing studies reporting the effects of pemafibrate in patients with NAFLD are limited to shortterm outcomes $[6,7]$. There are no reports of long-term outcomes in the literature at this time. The aim of this study is to evaluate the long-term outcomes of patients with NAFLD treated with pemafibrate.

\section{Material and methods}

\section{Study population}

This is a retrospective observational study which enrolled non-diabetic patients with NAFLD complicated by dyslipidemia treated with pemafibrate between June 2019 and October 2020. Their medical records were retrospectively reviewed, and data were abstracted. The inclusion criteria include: 1) fatty liver diagnosed by abdominal ultrasound, 2) dyslipidemia treated with pemafibrate, 3 ) sustained elevation of alanine aminotransferase (ALT) $>30$ for more than three months before starting pemafibrate, 4) negative hepatitis $B$ surface antigen and hepatitis $C$ virus antibody tests, 5) normal serum immunoglobulin $\mathrm{G}$ level, 6) alcohol consumption $<30 \mathrm{~g} /$ day in males and $<20 \mathrm{~g} /$ day in females [5]. The exclusion criteria include: 1) severe chronic kidney disease (serum creatinine $>2.5 \mathrm{mg} / \mathrm{dl}$ ), 2) history of previous pemafibrate use, 3 ) patients who stopped pemafibrate within 12 months or 4) patients with diabetes mellitus. The Institutional Review Board approved this retrospective review.

\section{Evaluation of hepatic inflammation, function and fibrosis}

Hepatic inflammation was assessed using ALT level, which is a validated marker to evaluate the progression of hepatic fibrosis in patients with NASH $[8,9]$. Hepatic function was evaluated using the albumin-bilirubin (ALBI) score calculated using the serum albumin and total bilirubin levels [10]. The ALBI score is proportionate to the indocyanine green retention test at 15 min (ICG-R15) [11]. Hepatic fibrosis was assessed by the cutoff index of the serum Mac-2 binding protein glucosylation isomer (M2BPGi) that reflects the grade of hepatic fibrosis without the need to calculate multiple factors. The FIB-4 index was also determined to estimate hepatic fibrosis $[12,13]$.

\section{Statistical analysis}

All statistical analyses were performed using StatFlex 7.0 software (Artech Co., Ltd., Osaka, Japan). Changes in parameters during one year of therapy were assessed with the Wilcoxon rank-sum test. To evaluate the association between parameters, Spearman's rank correlation coefficient was used.

\section{Results}

\section{Baseline characteristics}

Twenty-eight patients with NAFLD received pemafibrate and six patients were excluded for the following reasons: lost to follow-up $(n=4)$ and lack of critical data $(n=2)$. Finally, the remaining 22 patients were included in this study. Pemafibrate $0.1 \mathrm{mg}$ twice daily was given and there were no dose escalations. For the treatment of dyslipidemia, 19 patients (86\%) were previously treated with a statin for more than six months and pemafibrate was given in addition to the statin (Table 1). We confirmed normal fasting plasma glucose levels and $\mathrm{HbA}_{1 \mathrm{c}}$ level $<6.5 \%$ without diabetic medication in all patients before starting pemafibrate. There was no combination use of ursodeoxycholic acid or tocopherol. There were no adverse events during the study period.

\section{Changes in hepatic markers and lipid profiles}

We evaluated changes in parameters during one year of pemafibrate therapy (Table 2). Parameters that changed significantly at one year are shown in Figs. 1 and 2. As markers of hepatic inflammation, ALT significantly decreased during the first three months of therapy and was maintained. Alkaline phosphatase (ALP) decreased in all patients. Low-density lipoprotein (LDL) and triglycerides decreased significantly at three months and were maintained. Regarding markers of hepatic function, the ALBI score significantly 
Table 1. Baseline characteristics of patients

\begin{tabular}{lc}
\hline Variable & $N=22$ \\
\hline Age (years), mean \pm SE & $60.4 \pm 2.5$ \\
\hline Gender (male), $n(\%)$ & $12(54)$ \\
\hline Current smoker, $n(\%)$ & $1(5)$ \\
\hline Complications treated with medications, $n(\%)$ & \\
\hline Hypertension & $8(36)$ \\
\hline Gastroesophageal reflux disease & $9(41)$ \\
\hline Diabetes mellitus & $0(0)$ \\
\hline Combination use, $n$ (\%) & $19(86)$ \\
\hline Statins & $5(23)$ \\
\hline Ezetimibe & $4(18)$ \\
\hline Angiotensin II receptor blockers & $1(5)$ \\
\hline Eicosapentaenoic acid/docosahexaenoic acid & \\
\hline SE - standard error
\end{tabular}

decreased during one year of therapy due to significantly elevated serum albumin and slightly decreased total bilirubin levels. As a marker of fibrosis, M2BPGi significantly decreased and was maintained for one year and platelet count increased significantly.

\section{Correlation analysis}

We then performed correlation analyses between M2BPGi and other parameters (Table 3). Changes in aspartate aminotransferase (AST), ALT and triglycerides showed strong positive correlations with changes in M2BPGi. Control of hepatic inflammation and dyslipidemia was associated with the improvement in hepatic fibrosis.

\section{Discussion}

Cirrhosis and subsequent hepatocellular carcinoma are life-threatening diseases. Cirrhosis is caused by persistent hepatitis. Chronic viral hepatitis is becoming a controllable disease with advances in antiviral therapy. NAFLD and NASH are important in the pathogenesis of chronic hepatitis. This study shows that one year of continuous pemafibrate therapy on NAFLD durably decreases ALT levels, a reliable marker of hepatic inflammation. Interestingly, long-term treatment also improves markers of hepatic function and fibrosis such as the ALBI score and M2BPGi.

We previously reported short-term improvement of hepatic inflammation in non-diabetic patients with NAFLD treated with pemafibrate $0.1 \mathrm{mg}$ twice daily for three months [5]. In a phase III trial, pemafibrate significantly decreased ALT and $\gamma$-glutamyl transpep-
Table 2. Changes in clinical parameters after a one-year course of pemafibrate therapy

\begin{tabular}{lccc}
\hline Parameter & Baseline & One year & $P$-value \\
\hline Weight $(\mathrm{kg})$, mean \pm SE & $70.4 \pm 3.1$ & $69.9 \pm 3.2$ & 0.088 \\
\hline Body mass index & $27.0 \pm 0.7$ & $26.8 \pm 0.7$ & 0.081 \\
\hline AST $(\mathrm{U} / \mathrm{l})$ & $53.4 \pm 6.0$ & $35.1 \pm 1.7$ & $<0.001$ \\
\hline ALT $(\mathrm{U} / \mathrm{l})$ & $64.7 \pm 5.7$ & $29.7 \pm 2.5$ & $<0.001$ \\
\hline ALP $(\mathrm{U} / \mathrm{l})$ & $291.6 \pm 25.3$ & $170.0 \pm 10.1$ & $<0.001$ \\
\hline$\gamma$-GTP $(\mathrm{U} / \mathrm{l})$ & $87.6 \pm 18.8$ & $42.9 \pm 12.1$ & $<0.001$ \\
\hline Platelet count $\left(\times 10^{4} / \mathrm{\mu l}\right)$ & $24.1 \pm 0.8$ & $26.0 \pm 1.0$ & 0.007 \\
\hline Estimated GFR & $75.8 \pm 3.4$ & $74.5 \pm 3.5$ & 0.165 \\
(ml/min/1.73 m²) & & & \\
\hline LDL cholesterol (mg/dl) & $98.3 \pm 6.3$ & $82.3 \pm 3.9$ & 0.002 \\
\hline HDL cholesterol (mg/dl) & $54.0 \pm 2.8$ & $54.8 \pm 2.7$ & 0.435 \\
\hline Triglyceride $(\mathrm{mg} / \mathrm{dl})$ & $125.9 \pm 14.2$ & $74.3 \pm 6.9$ & $<0.001$ \\
\hline Uric acid $(\mathrm{mg} / \mathrm{dl})$ & $5.6 \pm 0.1$ & $5.6 \pm 0.3$ & 0.083 \\
\hline Total bilirubin $(\mathrm{mg} / \mathrm{dl})$ & $1.0 \pm 0.1$ & $0.8 \pm 0.1$ & 0.003 \\
\hline Serum albumin (g/dl) & $4.3 \pm 0.1$ & $4.6 \pm 0.1$ & $<0.001$ \\
\hline ALBl score & $-2.8 \pm 0.1$ & $-3.1 \pm 0.1$ & $<0.001$ \\
\hline FIB-4 index & $1.1 \pm 0.2$ & $0.9 \pm 0.2$ & 0.448 \\
\hline M2BPGi & $0.9 \pm 0.1$ & $0.7 \pm 0.1$ & 0.003 \\
\hline SE - sandad & & &
\end{tabular}

SE - standard error, AST - aspartate aminotransferase, ALT - alanine aminotransferase, ALP - alkaline phosphatase, $\gamma$-GTP - $\gamma$-glutamyl transpeptidase, GFR - glomerular filtration rate, LDL - low-density lipoprotein, $H D L$ - high-density lipoprotein, $A L B I$ - albumin-bilirubin, M2BPGi - Mac-2 binding protein glucosylation isomer

tidase ( $\gamma$-GTP) levels but about half of patients continued to use alcohol and the diagnosis of fatty liver was not established [14]. Ikeda et al. also reported that 17 patients with NAFLD treated with pemafibrate for six months showed decreases in ALT and $\gamma$-GTP levels. A recent Japanese single-arm prospective study for 12 weeks of pemafibrate therapy reported on 20 patients with NAFLD and showed significant reductions of AST, ALT and $\gamma$-GTP and an elevation of platelet count [6]. These three short-term studies did not demonstrate significant improvement in markers of hepatic fibrosis. Similar to atherosclerotic changes, the development of hepatic fibrosis takes place over a long time. It may take a longer treatment period to observe improvement in liver function in patients with NAFLD. This study is the first to show long-term improvement in hepatic profiles in patients with NAFLD treated with pemafibrate.

Pemafibrate is a selective PPAR- $\alpha$ agonist. PPAR- $\alpha$ knockout mice develop hepatic inflammation and steatosis [15]. Patients with NASH show low levels of expression of PPAR- $\alpha$ mRNA in the liver [16]. We proposed that pemafibrate may have a therapeutic effect on hepatic inflammation in our previous study [5]. 
A

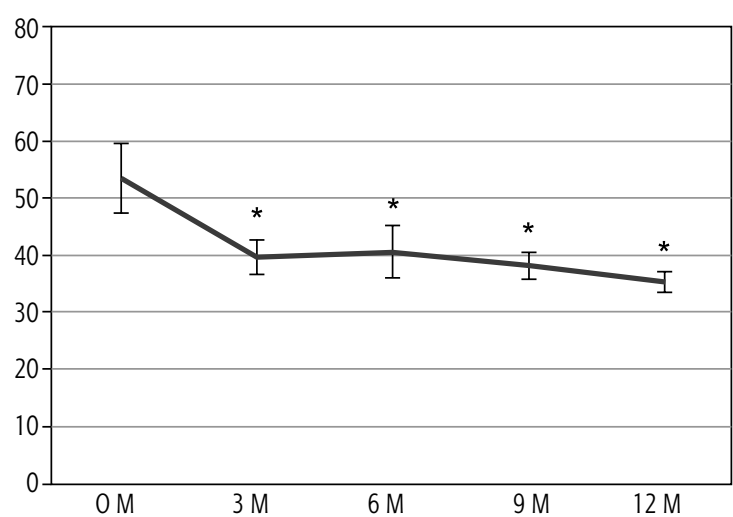

C

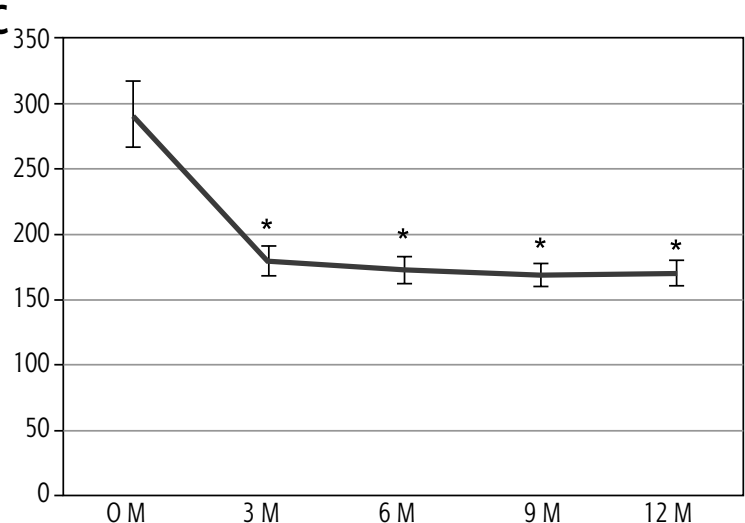

$\mathrm{E}$

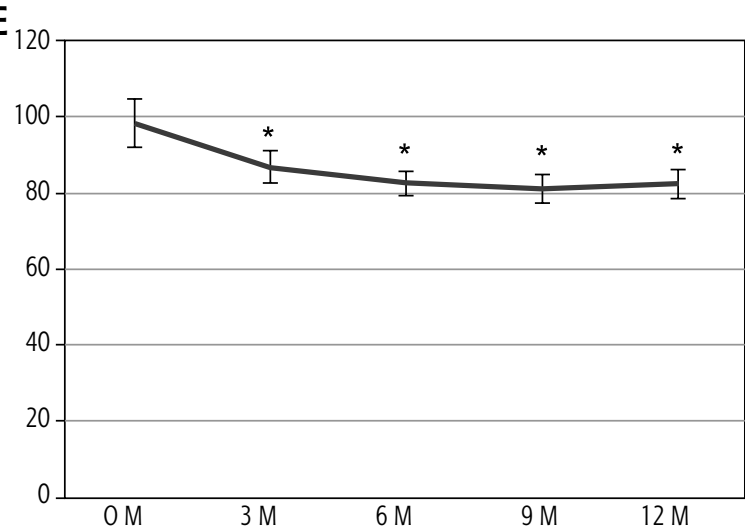

B

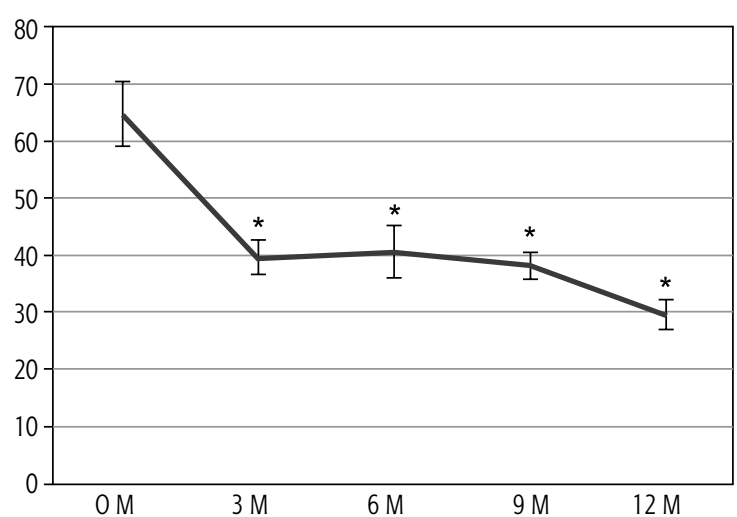

D

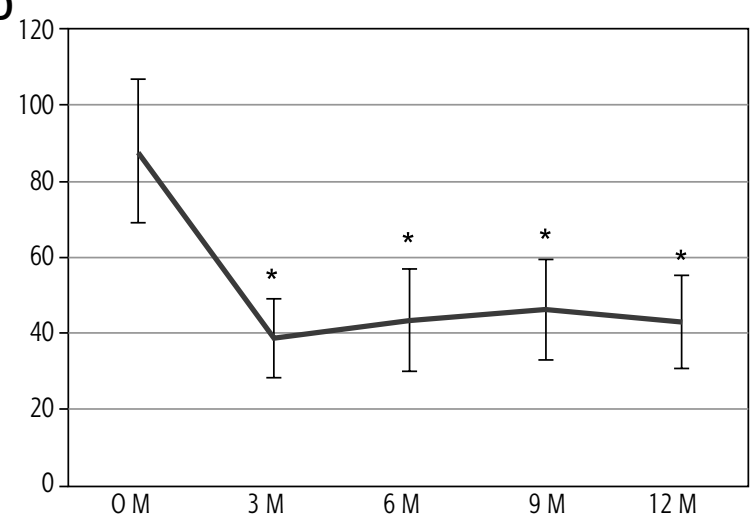

$\mathbf{F}$

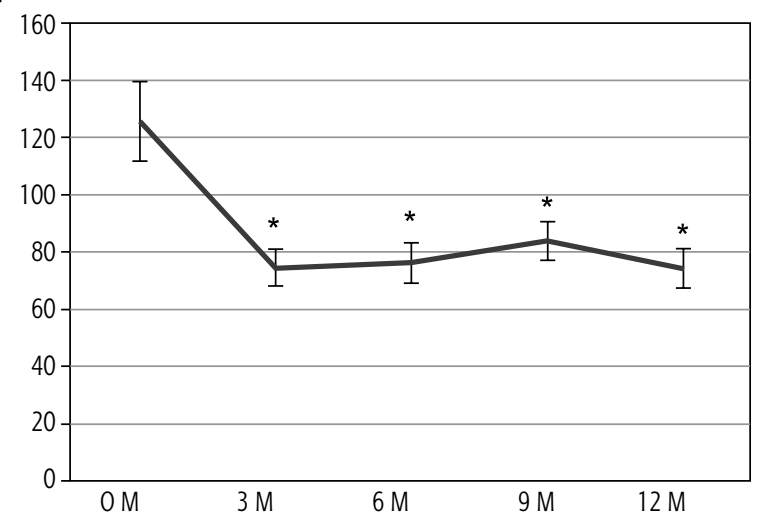

Fig. 1. Changes in hepatobiliary and lipid parameters show significant changes after one year of pemafibrate therapy. A) Aspartate aminotransferase (AST), B) alanine aminotransferase (ALT), C) alkaline phosphatase, D) $\gamma$-glutamyl transpeptidase ( $\gamma$-GTP), E) low-density lipoprotein (LDL) cholesterol and F) triglyceride. ${ }^{*} p<0.05$ compared to baseline

A durable reduction of ALT is associated with histological amelioration of NASH [9]. In the present study, we revealed that a reduction of M2BPGi observed in patients using pemafibrate for one year correlates significantly with a reduction of ALT. This result suggests that pemafibrate ameliorates hepatic fibrosis due to suppression of hepatic inflammation through the PPAR-a signal.

Regarding lipid profiles, the reduction of serum triglycerides also correlates with a reduction in M2B-
PGi. We cannot determine whether this correlation is a causal relationship or not. PPAR- $\alpha$ upregulated by pemafibrate promotes production of lipoprotein lipase and apolipoprotein, resulting in the reduction of serum triglyceride levels [17]. Regarding hepatic involvement, Sasaki et al. reported an improvement in NAFLD activity score without affecting triglyceride accumulation in the liver in a murine model of NASH [3]. We assume that the improvement in hepatic fibrosis and serum triglycerides may not directly interact, 

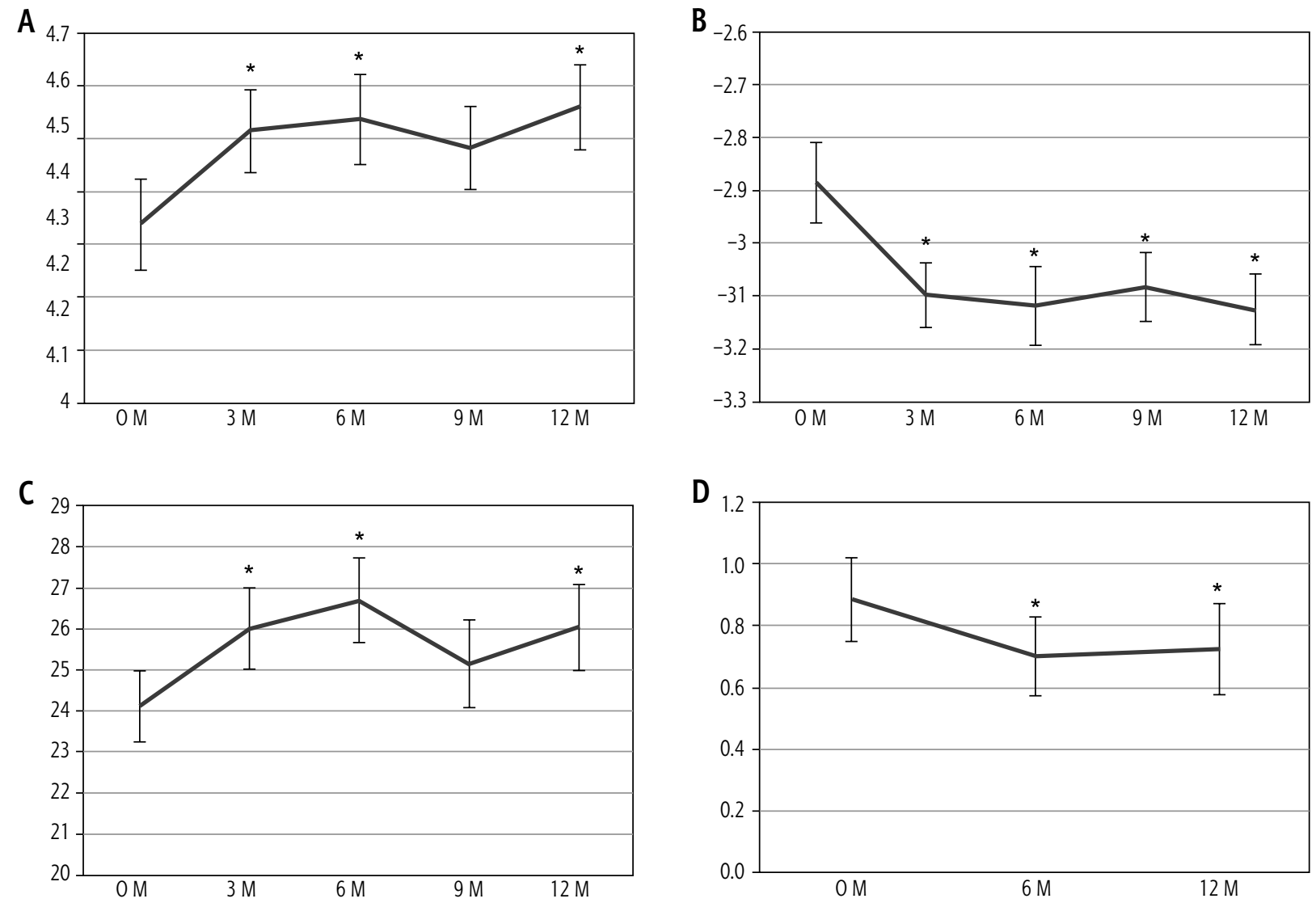

Fig. 2. Changes in parameters regarding hepatic function and fibrosis show significant changes after one year of pemafibrate therapy. A) Serum albumin, B) albumin-bilirubin (ALBI) score, C) platelet count and D) Mac-2 binding protein glucosylation isomer (M2BPGi). * $p<0.05$ compared to baseline

but a reduction of triglycerides may be a marker of improved liver function.

The high selectivity of pemafibrate for PPAR- $\alpha$ is the key to explain improved hepatic function. Although fenofibrate use results in elevated levels of AST, ALT and $\gamma$-GTP, pemafibrate significantly decreased them in a phase III trial [14]. Honda et al. reported that high-dose pemafibrate showed greater improvement of hepatic inflammation and fibrosis than fenofibrate in a rodent model of NASH [18]. The high selectivity for PPAR- $\alpha$ by pemafibrate may contribute to the observed improvement in hepatic fibrosis.

Elevations of platelet and albumin levels were observed in this study. It is well known that a decreased platelet count is associated with the degree of hepatic fibrosis in NAFLD [19]. The significant increase in platelet count shown in this study may reflect an improvement in hepatic inflammation with reduced secretion of thrombopoietin and hypersplenism [20]. Albumin is synthesized in the liver, and improved hepatic inflammation after pemafibrate therapy may allow recovered production of albumin. Weight reduction is effective to improve NAFLD, but this study did not show significant weight reduction after one year of therapy. Hence, improved hepatic and lipid profiles are thought to be due to enhanced activation of PPAR- $\alpha$ activation.

There are some acknowledged limitations to this study. First, this is a single-center retrospective observational study without a placebo group. Second, there was no histopathological evaluation of the liver before and after treatment. Third, combination use with statins in some patients may bias the results. There are some advantages to this study. First, the treatment period with pemafibrate on NAFLD in this study is longer than in past studies, which had 12-24 weeks of follow-up $[6,7]$. Second, no patients with diabetes were included. The development of NAFLD is influenced by concurrent type 2 diabetes [1], and some anti-diabetic medications such as sodium-glucose cotransporter-2 inhibitors could ameliorate NAFLD [21].

In conclusion, long-term pemafibrate therapy improves markers of hepatic inflammation, function and fibrosis in non-diabetic patients with NAFLD. Improvement of hepatic fibrosis markers significantly correlates with improved hepatic inflammation markers and triglyceride levels. A randomized-controlled long-term study is necessary to confirm these results. 
Table 3. Association of changes in M2BPGi with other parameters

\begin{tabular}{|c|c|c|}
\hline \multirow{2}{*}{$\begin{array}{l}\text { Parameters changed during } \\
\text { treatment }\end{array}$} & $\triangle \mathrm{M} 2 \mathrm{BPGi}$ & \multirow[t]{2}{*}{$P$-value } \\
\hline & $\begin{array}{l}\text { Correlation } \\
\text { coefficient }\end{array}$ & \\
\hline$\Delta$ Weight (kg) & 0.044 & 0.843 \\
\hline$\triangle \mathrm{AST}(\mathrm{U} / \mathrm{l})$ & 0.716 & $<0.001$ \\
\hline$\Delta \mathrm{ALT}(\mathrm{U} / \mathrm{l})$ & 0.648 & 0.001 \\
\hline$\triangle \mathrm{ALP}(\mathrm{U} / \mathrm{l})$ & -0.059 & 0.791 \\
\hline$\Delta \gamma-\mathrm{GTP}(\mathrm{U} / \mathrm{l})$ & 0.228 & 0.305 \\
\hline$\Delta$ Platelet count $\left(\times 10^{4} / \mu \mathrm{l}\right)$ & -0.041 & 0.855 \\
\hline$\Delta$ Estimated GFR $\left(\mathrm{ml} / \mathrm{min} / 1.73 \mathrm{~m}^{2}\right)$ & 0.129 & 0.564 \\
\hline$\Delta \mathrm{LDL}$ cholesterol (mg/dl) & 0.249 & 0.263 \\
\hline$\Delta \mathrm{HDL}$ cholesterol (mg/dl) & -0.422 & 0.050 \\
\hline$\Delta$ Triglyceride (mg/dl) & 0.524 & 0.012 \\
\hline$\Delta$ Uric acid (mg/dl) & 0.178 & 0.428 \\
\hline$\Delta$ Total bilirubin (mg/dl) & 0.125 & 0.576 \\
\hline$\Delta$ Serum albumin $(\mathrm{g} / \mathrm{dl})$ & -0.237 & 0.287 \\
\hline$\triangle \mathrm{ALBI}$ score & 0.251 & 0.259 \\
\hline$\Delta$ FIB-4 index & 0.490 & 0.020 \\
\hline
\end{tabular}

M2BPG - Mac-2 binding protein glucosylation isomer, AST - aspartate aminotransferase, ALT - alanine aminotransferase, ALP - alkaline phosphatase, $\gamma$-GTP - $\gamma$-glutamyl transpeptidase, GFR - glomerular filtration rate, LDL - low-density lipoprotein, HDL - highdensity lipoprotein, $A L B I$ - albumin-bilirubin. Values of $p<0.05$ are shown in bold font.

\section{Disclosure}

S.S. has received honoraria from AstraZeneca, Nippon Boehringer Ingelheim Co., Daiichi Sankyo Co., Kowa Co., Mitsubishi Tanabe Pharma Co., Otsuka Pharmaceutical Co. and Takeda Pharmaceutical Co., Ltd. M.O. received a research support from Takeda Pharmaceutical Co., Ltd. M.O. also received honoraria from Astellas Pharma Inc, AstraZeneca, Nippon Boehringer Ingelheim Co., Daiichi Sankyo Co., Eli Lilly and Co., Kyowa Hakko Kirin Co., Kowa Co., Merck and Co., Novo Nordisk Pharma, Taisho Pharmaceutical Co., Mitsubishi Tanabe Pharma Co., ONO PHARMACEUTICAL CO., Sanofi and Takeda Pharmaceutical Co., Ltd. Other authors declare no conflict of interest.

\section{References}

1. Watanabe S, Hashimoto E, Ikejima K, et al. Evidence-based clinical practice guidelines for nonalcoholic fatty liver disease/ nonalcoholic steatohepatitis. J Gastroenterol 2015; 50: 364-377.

2. Chalasani N, Younossi Z, Lavine JE, et al. The diagnosis and management of nonalcoholic fatty liver disease: Practice guidance from the American Association for the Study of Liver Diseases. Hepatology 2018; 67: 328-357.

3. Sasaki Y, Asahiyama M, Tanaka T, et al. Pemafibrate, a selective PPAR $\alpha$ modulator, prevents non-alcoholic steatohepatitis development without reducing the hepatic triglyceride content. Sci Rep 2020; 10: 7818.

4. Ishibashi S, Yamashita S, Arai H, et al. Effects of K-877, a novel selective PPARalpha modulator (SPPARMalpha), in dyslipidaemic patients: A randomized, double blind, active- and placebocontrolled, phase 2 trial. Atherosclerosis 2016; 249: 36-43.

5. Shinozaki S, Tahara T, Lefor AK, Ogura M. Pemafibrate decreases markers of hepatic inflammation in patients with non-alcoholic fatty liver disease. Clin Exp Hepatol 2020; 6: 270-274.

6. Seko Y, Yamaguchi K, Umemura A, et al. Effect of pemafibrate on fatty acid levels and liver enzymes in non-alcoholic fatty liver disease patients with dyslipidemia: A single-arm, pilot study. Hepatol Res 2020; 50: 1328-1336.

7. Ikeda S, Sugihara T, Hoshino Y, et al. Pemafibrate dramatically ameliorated the values of liver function tests and fibrosis marker in patients with non-alcoholic fatty liver disease. Yonago Acta Med 2020; 63: 188-197.

8. Ekstedt M, Franzen LE, Mathiesen UL, et al. Long-term follow-up of patients with NAFLD and elevated liver enzymes. Hepatology 2006; 44: 865-873.

9. Seko Y, Sumida Y, Tanaka S, et al. Serum alanine aminotransferase predicts the histological course of non-alcoholic steatohepatitis in Japanese patients. Hepatol Res 2015; 45: E53-61.

10. Johnson PJ, Berhane S, Kagebayashi C, et al. Assessment of liver function in patients with hepatocellular carcinoma: a new evidence-based approach-the ALBI grade. J Clin Oncol 2015; 33: 550-558.

11. Hiraoka A, Kumada T, Kudo M, et al. Albumin-bilirubin (ALBI) grade as part of the evidence-based clinical practice guideline for HCC of the Japan Society of Hepatology: a comparison with the liver damage and Child-Pugh classifications. Liver Cancer 2017; 6: 204-215.

12. Angulo P, Hui JM, Marchesini G, et al. The NAFLD fibrosis score: a noninvasive system that identifies liver fibrosis in patients with NAFLD. Hepatology 2007; 45: 846-854.

13. Sterling RK, Lissen E, Clumeck N, et al. Development of a simple noninvasive index to predict significant fibrosis in patients with HIV/HCV coinfection. Hepatology 2006; 43: 1317-1325.

14. Ishibashi $\mathrm{S}$, Arai $\mathrm{H}$, Yokote $\mathrm{K}$, et al. Efficacy and safety of pemafibrate (K-877), a selective peroxisome proliferator-activated receptor a modulator, in patients with dyslipidemia: Results from a 24-week, randomized, double blind, active-controlled, phase 3 trial. J Clin Lipidol 2018; 12: 173-184.

15. Stienstra R, Mandard SP, Patsouris D, et al. Peroxisome proliferator-activated receptor a protects against obesity-induced hepatic inflammation. Endocrinology 2007; 148: 2753-2763.

16. Fujita K, Nozaki Y, Wada K, et al. Dysfunctional very-low-density lipoprotein synthesis and release is a key factor in nonalcoholic steatohepatitis pathogenesis. Hepatology 2009; 50: 772 780 .

17. Yamashita S, Masuda D, Matsuzawa Y. Pemafibrate, a new selective PPARa modulator: drug concept and its clinical applications for dyslipidemia and metabolic diseases. Curr Atheroscler Rep 2020; 22: 5.

18. Honda Y, Kessoku T, Ogawa Y, et al. Pemafibrate, a novel selective peroxisome proliferator-activated receptor alpha modulator, improves the pathogenesis in a rodent model of nonalcoholic steatohepatitis. Sci Rep 2017; 7: 42477.

19. Yoneda M, Fujii H, Sumida Y, et al. Platelet count for predicting fibrosis in nonalcoholic fatty liver disease. J Gastroenterol 2011; 46: 1300-1306. 